\title{
ON WEITZENBÖCK'S THEOREM IN POSITIVE CHARACTERISTIC
}

\author{
A. FAUNTLEROY
}

\begin{abstract}
ABSTRACr. Let $k$ be an algebraically closed field and let $f: \dot{G}_{a} \rightarrow$ GL $(V)$ be a finite-dimensional $k$-rational representation of the additive group $G_{a}$. If the subspace of $G_{a}$-fixed points in $V$ is a hyperplane, then the ring of $G_{a}$-invariant polynomial functions on $V$ is finitely generated over $k$. This result is an analog of a classical theorem of Weitzenböck, a modern proof of which has been given by $\mathrm{C}$. S. Seshadri.
\end{abstract}

Introduction. Let $k$ be an algebraically closed field and $G_{a}$ the one-dimensional vector group over $k$. Let $G_{a}$ act linearly on the finite-dimensional $k$-vector space $V$ so that the subspace of fixed points on $V$ has codimension one. The purpose of this note is to show that, in this case, the ring of $G_{a}$-invariant polynomial functions on $V$ is finitely generated over $k$. This result is an analog of a classical result of Weitzenböck, a modern proof of which is due to C. S. Seshadri [6].

If a rational representation of $G_{a}$ on $V$ factors through an $\operatorname{SL}(2, k)$ representation, then the representation is called fundamental. Seshadri [6] gives a proof of Weizenböck's theorem for fundamental representations and shows that every representation of $G_{a}$ in characteristic zero is fundamental. An example is given here which shows that this is not the case in positive characteristics. In particular, there exist representations of $G_{a}$ having fixed point loci of codimension one which are not fundamental.

Notations and conventions. Throughout, $k$ denotes a fixed algebraically closed field of arbitrary characteristic. All algebraic groups are affine $k$ groups, all varieties and morphisms are defined over $k$, and representations of algebraic groups are assumed $k$-rational. A point of a variety is always a $k$-rational point.

Let $\rho: G_{a} \rightarrow \mathrm{GL}(V)$ be a given representation; i.e., a homomorphism of algebraic groups. Give $V$ the structure of an affine space with ring of functions $S\left(V^{*}\right)$-the symmetric algebra on the $k$-dual of $V$. The set $V_{0}$ of $G_{a}$-fixed points of $V$ is a linear subspace of $V$. Suppose that $V_{0}$ has codimension one in $V$. Let $\left\{e_{1}, \ldots, e_{n}\right\}$ be a basis of $V$ such that $\left\langle e_{1}, \ldots, e_{n-1}\right\rangle=$ $V_{0}$. Let $\left\{x_{1}, \ldots, x_{n}\right\}$ be the corresponding dual basis. Then $S\left(V^{*}\right) \cong$

Received by the editors February 17, 1976 and, in revised form, May 26, 1976 and September 21, 1976.

AMS (MOS) subject classifications (1970). Primary 15M05, 14M10, $14 \mathrm{~N} 10$.

Key words and phrases. $G_{a}$-actions, ring of invariants. 
$k\left[x_{1}, \ldots, x_{n}\right]$ and, since $G_{a}$ must act trivially on $V / V_{0}$, the canonical action of $G_{a}$ on $k\left[x_{1}, \ldots, x_{n}\right]$ is as follows:

$$
\begin{aligned}
& t \cdot x_{i}=x_{i}+a_{i}(t) x_{n}, \quad 1 \leqslant i<n-1, \\
& t \cdot x_{n}=x_{n}, \quad \text { all } t \in G_{a} .
\end{aligned}
$$

Here $a_{i}(T) \in k\left[G_{a}\right]=k[T]$ is an additive function on $G_{a}$, so it is a $p$-polynomial in $k[T]$ if $p=$ char $k>0$. The following lemma is well known, but we give a proof for lack of a precise reference.

LemMa 1. Let $A \subseteq B$ be integral domains with $A$ normal and $B$ integral over $A$. Let $E$ be $a$ set of ring endomorphisms of $B$. Denote by $A^{E}$ and $B^{E}$ the subring of $E$-invariant elements of $A$ and $B$, respectively. Then $B^{E}$ is integral over $A^{E}$.

Proof. Let $b \in B^{E}$ and let $\underline{P}(T)=T^{m}+a_{i} T^{m-1}+\cdots+a_{m}$ be the minimal monic polynomial of $b$ over the quotient field of $A$. Since $A$ is normal, all the $a_{i}$ belong to $A$. Now, if $e \in E$, then

$$
0=e(P(b))=b^{m}+e\left(a_{2}\right) b^{m-1}+\cdots+e\left(a_{m}\right)
$$

and hence, $a_{i}=e\left(a_{1}\right), \ldots, a_{m}=e\left(a_{m}\right)$.

Suppose char $k=p>0$. Let $D$ be the noncommutative ring of $p$-polynomials over $k$ (i.e., composition of functions as the law of multiplication). It is well known (cf. [4] or [5, Theorem 1]) that $D$ is a right (and left) Euclidean domain. Consequently, every nonzero left ideal is principal and of finite codimension as a $k$-module. It follows that if $a$ and $b$ are nonzero elements of $D$, then $D a \cap D b \neq\{0\}$ and, hence, $a$ and $b$ have a nonzero left least common multiple.

We are now prepared to prove our main result.

THEOREM. Let $V$ be a finite-dimensional rational $G_{a}$-module and suppose the et of fixed points in $V$ has codimension one in $V$. Then the ring of $G_{a}$-invariant polynomial functions on $V$ is finitely generated over $k$.

Proof. We need only consider the case char $k=p>0$. We may assume $k[V]=k\left[x_{1}, \ldots, x_{n}\right]$ where the coordinate functions $x_{1}, \ldots, x_{n}$ satisfy (1). Note that, by a simple change of basis of $V_{0}$, we may assume that the degrees of all the $a_{i}(T)$ in (1) are equal. By the preceding remarks, we know that for each $i$ there exist a pair of nonzero $p$-polynomials $\left(b_{i}, d_{i}\right)$ such that $b_{i} \circ a_{1}=$ $d_{i} \circ a_{i}$. For $2<i \leqslant n-1$ set

$$
z_{i}=d_{i}\left(x_{i} / x_{n}\right)-b_{i}\left(x_{1} / x_{n}\right)
$$

It is easy to verify that $z_{i} \in k\left(x_{1}, \ldots, x_{n}\right)^{G_{a}}$. Since the degree of $a_{1}$ equals the degree of $a_{i}$, the degrees of $b_{i}$ and $d_{i}$ must be the same for any given $i$. If this degree is $p^{r_{i}}$, then multiplying (2) by $x_{n}^{p^{\prime \prime}}$ gives the invariant polynomial 


$$
y_{i}=x_{i}^{p_{i}^{\prime}}+\sum_{j=0}^{r_{i}-1} r_{i j}\left(x_{1}, x_{n}\right) x_{i}^{p^{j}}, \quad 2<i<n-1,
$$

where $r_{i j}\left(x_{1}, x_{n}\right) \in k\left[x_{1}, x_{n}\right]$.

It follows from (3) that $k\left[x_{1}, \ldots, x_{n}\right]$ is integral over

$$
S=k\left[y_{2}, \ldots, y_{n-1}, x_{1}, x_{n}\right] \text {. }
$$

Moreover, $S$ is a polynomial ring over $k$, hence, $S$ is normal. Now $k\left[x_{1}, \ldots, x_{n}\right]^{G_{a}}$ is integrally closed and by Lemma 1 , integral over $S^{G_{a}}$. But $S=k\left[y_{1}, \ldots, y_{n-1} x_{n}\right]\left[x_{1}\right]$ and, since $y_{2}, \ldots, y_{n-1}$ and $x_{n}$ are invariants and $k$ is infinite, it follows that

$$
S^{G_{a}}=k\left[y_{1}, \ldots, y_{n-1}, x_{n}\right] .
$$

By [7, p. 267, Theorem 9], $k\left[x_{1}, \ldots, x_{n}\right]^{G_{a}}$ is finitely generated over $k$. Q.E.D.

We show now that the theorem does in fact give an extension of Seshadri's result. Assume that $p=$ char $k>2$. Let $x, y$ and $z$ be coordinates on $V_{0}=k^{3}$ and let $G_{a}$ act on $V_{0}$ via the assignments:

$$
t \cdot x=x+t^{p}, \quad t \cdot y=y+t z, \quad t \cdot z=z \quad \text { all } t \in G_{a} .
$$

Note that $z=0$ defines the fixed point locus on $V_{0}$. Moreover, if $a x+b y+$ $c z=0$ is a $G_{a}$-stable hyperplane, then $a t^{p}+b t+c=c$, all $t \in G_{a}$. Hence, $a=b=0$ and so $z=0$ is the unique $G_{a}$-stable hyperplane in $V_{0}$.

We claim that $V_{0}$ is not a fundamental $G_{a}$-module. We need the following lemma.

LEMMA 2. Suppose char $k=p>2$. Then every 3-dimensional rational $\mathrm{SL}(2, k)$-module is completely reducible.

Proof. Let $V$ be a 3-dimensional rational SL(2,k)-module. If $V$ is simple or trivial there is nothing to prove. Since $V$ is completely reducible if and only if $V^{*}$ is, we may assume that $V$ contains a 2-dimensional nontrivial submodule $W$. Then $W$ is necessarily irreducible and the action of $\operatorname{SL}(2, k)$ on $W$ is given by an $i$ th iterate of the Frobenius map composed with the identity representation (cf. [1]). Let $\sigma=-\operatorname{Id} \in \operatorname{SL}(2, k)$. Then $\sigma$ is represented by the matrix

$$
M_{\sigma}=\left[\begin{array}{ccc}
-1 & 0 & \alpha \\
0 & -1 & \beta \\
0 & 0 & 1
\end{array}\right]
$$

with respect to some basis of $V$. Since the minimal polynomial for $\boldsymbol{M}_{\boldsymbol{\sigma}}$ is $T^{2}-1, M_{\sigma}$ is diagonalizable, so it is represented by

$$
M^{\prime}=\left[\begin{array}{ccc}
-1 & 0 & 0 \\
0 & -1 & 0 \\
0 & 0 & 1
\end{array}\right]
$$

in a suitable basis of $V$. But $\sigma$ lies in the centre of $\operatorname{SL}(2, k)$ and the centralizer of $M^{\prime}$ in $\operatorname{GL}(3, k)$ is 


$$
\left\{\left[\begin{array}{cc}
A & 0 \\
0 & *
\end{array}\right], A \in \mathrm{GL}(2, k)\right\} .
$$

Thus $V \wedge W \oplus k$ and the lemma is proved.

Now if $V$ is a 3-dimensional fundamental $G_{a}$-module, then either $V$ is irreducible as an $\operatorname{SL}(2, k)$-module, or $V \cong W \oplus k$ as an $\operatorname{SL}(2, k)$-module, where $W$ is a nontrivial $\operatorname{SL}(2, k)$-module of dimension 2 . In the first case, we see, by [1], that $V$ is isomorphic to an interate of the Frobenius composed with the irreducible representation of $\operatorname{SL}(2, k)$ on the space of forms of degree two in two variables. For this module the action of $G_{a}$ is given by the matrix representation

$$
t \rightarrow\left(\begin{array}{lll}
1 & 2 t^{p^{s}} & t^{2 p^{s}} \\
0 & 1 & t^{p^{s}} \\
0 & 0 & 1
\end{array}\right)
$$

It follows that the fixed point locus is a line, not a plane. In the second case, the action of $G_{a}$ is given by a matrix representation of the form

$$
t \rightarrow\left(\begin{array}{lll}
1 & t^{p^{r}} & 0 \\
0 & 1 & 0 \\
0 & 0 & 1
\end{array}\right)
$$

The fixed point locus for this action is, indeed, a hyperplane. However, there are infinitely many $G_{a}$-stable hyperplanes. It follows that, in any case, $V_{0}$ is not a fundamental $G_{a}$-module.

REMARKs. 1. Suppose that char $k=0$ and $G_{a}$ acts linearly on $V$ so that $V^{G_{a}}$ has codimension one in $V$. Then, using arguments entirely analogous to those given above, one can show that $k[V]^{G_{a}}$ is a polynomial algebra over $k$. This seems unlikely in positive characteristic, but at present we know of no counterexamples. In general, $k[V]^{G_{a}}$ is not a polynomial algebra [2].

2. It is known (cf. [2, Remark 7]) that, for fundamental $G_{a}$-actions, $k[V]^{G_{a}}$ is the coordinate ring of a rational variety; i.e. the quotient field of $k[V]^{G_{a}}$ is purely transcendental over $k$. Moreover, if char $k=0$, it follows from Seshadri's proof of Weitzenböck's theorem that $k[V]^{G_{a}}$ is actually the ring of invariants of an $\operatorname{SL}(2, k)$ action on a larger polynomial algebra; hence, the recent results of Hochster and Roberts [3] imply that, in this case, $k[V]^{G_{a}}$ is a Cohen-Macaulay ring. It would be interesting to know if either or both of these results hold in positive characteristic for the 'codimension one' actions discussed in this paper.

ACKNOWLEDGEMENT. The author is grateful to the referee of an earlier version of this manuscript for several simplifications and suggestions.

\section{REFERENCES}

1. A. Borel, Linear representations of semi-simple algebraic groups, Proc. Sympos. Pure Math., vol. 29, Amer. Math. Soc., Providence, R. I., 1975, pp. 421-440. 
2. A. Fauntleroy, Linear $G_{a}$-actions on affine spaces and associated rings of invariants, J. Pure Appl. Algebra 9 (1977).

3. M. Hochster and J. Roberts, Rings of invariants of reductive groups acting on regular rings are Cohen-Macaulay, Advances in Math. 13 (1974), 115-175.

4. T. Kambayashi, M. Miyanishi and M. Takeuchi, Unipotent algebraic groups, Lecture Notes in Math., vol. 414, Springer-Verlag, Berlin and New York, 1974.

5. O. Ore, On a special class of polynomials, Trans. Amer. Math. Soc. 35 (1933), 559-584.

6. C. S. Seshadri, On a theorem of Weitzenböck, J. Math. Kyoto 1-3 (1962).

7. Samuel Zariski, Commutative algebra, Vol. I, Van Nostrand, Princeton, N. J., 1960.

Departmant of Mathamatics, University of Illinois at Urbana-Champaign, Urbana, ILLNOIS 61801 